\title{
PROCESSING OF INFORMATION ENCODED IN COUPLED ONE-DIMENSIONAL MAPS
}

\author{
Alexander Yu. Loskutov and Valery M. Tereshko \\ Chair of Low-Temperature Physics, \\ Physics Faculty, \\ Moscow State University, \\ Moscow 119899, \\ RUSSIA \\ e-mail:khokh@mch.chem.msu.su
}

\begin{abstract}
We propose an analytical method of ciphering and deciphering various information by means of creation and destruction of new stable periodic orbits respectively, in parametrically coupled one-dimensional maps. This method is based on the following theorem.

Denote the set of values of the parameter corresponding to the chaotic behavior of map, by $\mathrm{C}$.

THEOREM [1]. There exists a subset $D$ of the set $C$ such that chaotic map with a cyclic parametric transformation generates stable cycles of a finite period.

The proof of this theorem is reduced to construction of multiple transformation maps and detection of certain parameter values at which iterations of such maps have stable periodic points.

Thus, parametric coupling of maps leads to creation of new periodic orbits of finite periods. Therefore, in a network of parametrically coupled maps a new information can be created. If the parameter values of maps belong to the chaotic set $C$, but they are outside $\mathrm{D}$, then all periodic orbits are "mixed", and information corresponding such orbits is hidden. However, when parameters are distributed in the subset D, the necessary information can be extracted. One may say that in this case the cipher key is the subset D. This subset $\mathrm{D}$ has extremely complex structure but, apparently, its Lebesgue measure is positive. Moreover, if in network the connection weights are small or approach zero then information given in initially created periodic orbits is destroyed.
\end{abstract}

\section{REFERENCES}

[1] Loskutov A.Yu. and Shishmarev A.I. (1992), Preprint N236 of The Max-Planck Society, Munich, Germany. 